\title{
Artificial Intelligence in the Fight Against COVID-19: Scoping Review
}

Alaa Abd-Alrazaq ${ }^{1}, \mathrm{PhD}$; Mohannad Alajlani ${ }^{2}, \mathrm{PhD}$; Dari Alhuwail ${ }^{3}, \mathrm{PhD}$; Jens Schneider ${ }^{1}$, PhD; Saif Al-Kuwari ${ }^{1}$, $\mathrm{PhD}$; Zubair Shah ${ }^{1}, \mathrm{PhD}$; Mounir Hamdi ${ }^{1}, \mathrm{PhD}$; Mowafa Househ ${ }^{1}, \mathrm{PhD}$

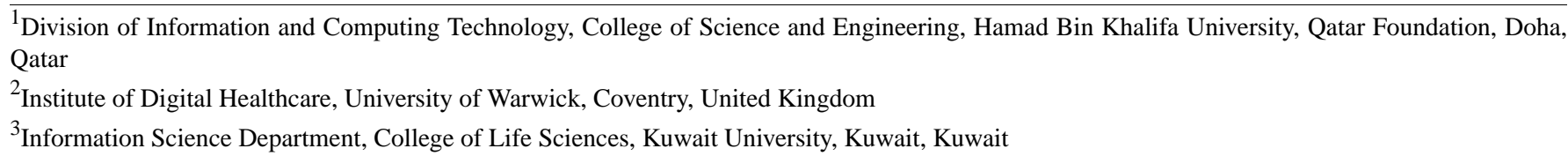

Corresponding Author:

Mowafa Househ, PhD

Division of Information and Computing Technology, College of Science and Engineering

Hamad Bin Khalifa University

Qatar Foundation

PO Box 5825, Doha Al Luqta St, Ar-Rayyan

Doha,

Qatar

Phone: 97455708549

Email: mhouseh@hbku.edu.qa

\section{Abstract}

Background: In December 2019, COVID-19 broke out in Wuhan, China, leading to national and international disruptions in health care, business, education, transportation, and nearly every aspect of our daily lives. Artificial intelligence (AI) has been leveraged amid the COVID-19 pandemic; however, little is known about its use for supporting public health efforts.

Objective: This scoping review aims to explore how AI technology is being used during the COVID-19 pandemic, as reported in the literature. Thus, it is the first review that describes and summarizes features of the identified AI techniques and data sets used for their development and validation.

Methods: A scoping review was conducted following the guidelines of PRISMA-ScR (Preferred Reporting Items for Systematic Reviews and Meta-Analyses Extension for Scoping Reviews). We searched the most commonly used electronic databases (eg, MEDLINE, EMBASE, and PsycInfo) between April 10 and 12, 2020. These terms were selected based on the target intervention (ie, AI) and the target disease (ie, COVID-19). Two reviewers independently conducted study selection and data extraction. A narrative approach was used to synthesize the extracted data.

Results: We considered 82 studies out of the 435 retrieved studies. The most common use of AI was diagnosing COVID-19 cases based on various indicators. AI was also employed in drug and vaccine discovery or repurposing and for assessing their safety. Further, the included studies used AI for forecasting the epidemic development of COVID-19 and predicting its potential hosts and reservoirs. Researchers used AI for patient outcome-related tasks such as assessing the severity of COVID-19, predicting mortality risk, its associated factors, and the length of hospital stay. AI was used for infodemiology to raise awareness to use water, sanitation, and hygiene. The most prominent AI technique used was convolutional neural network, followed by support vector machine.

Conclusions: The included studies showed that AI has the potential to fight against COVID-19. However, many of the proposed methods are not yet clinically accepted. Thus, the most rewarding research will be on methods promising value beyond COVID-19. More efforts are needed for developing standardized reporting protocols or guidelines for studies on AI.

(J Med Internet Res 2020;22(12):e20756) doi: 10.2196/20756

\section{KEYWORDS}

artificial intelligence; machine learning; deep learning; natural language processing; coronavirus; COVID-19; 2019-nCoV; SARS-CoV-2 


\section{Introduction}

\section{Background}

COVID-19 broke out in Wuhan, Hubei Province, China in December 2019 [1], spreading across the world and, as of May 2020 , claiming the lives of more than 330,000 people [2]. Caused by SARS-CoV-2, COVID-19 was declared a global pandemic by the World Health Organization in March 2020 [3]. Many individuals infected with COVID-19 experienced fever, dry cough, and fatigue; some faced a severe course of the medical condition, often requiring intensive care, including mechanical ventilation [4]. The contagious COVID-19 and its unprecedented volume of cases around the world have caused national and international disruptions to business, health care, education, transportation, and nearly every aspect of our daily lives [5]. Prompt and effective countermeasures are necessary to cap off the effects of this pandemic; comprehensive public health strategies that involve surveillance, diagnostics, clinical treatment, and research are required [6].

Leveraging digital tools and technologies to combat COVID-19 can augment public health strategies [7], for example, by using chatbots to address public inquiries about COVID-19. Additionally, using digital tools, public health professionals can track in real time the incidence of COVID-19 infections and potentially model their projection. Among such tools is artificial intelligence (AI) — a branch of computer science concerned with intelligently analyzing and handling complex information [8,9]-amplifying public health efforts against COVID-19. Despite the enthusiasm for AI applications since the 1950s, only recently have we witnessed interest in AI due to the availability of high-performance computing and vast amounts of data being generated every second [10].

AI enables machines to become intelligent, understand queries, sift through and connect mountains of data points, and draw actionable conclusions [11]. Although defining the taxonomy of AI is not trivial, its methods can be categorized based on the objective pursued: learn from knowledge, explore and discover knowledge, extract conclusions, and reason from knowledge [8].

Soon after the COVID-19 pandemic spread across the world, several governments, research institutes, and technology companies have issued calls to action urging researchers to develop AI applications to assist with COVID-19-related research [12]. From a hierarchical perspective, AI can support COVID-19 at different levels: the molecular level (eg, drug and vaccine discovery), patient level (eg, patient diagnosis), and population level (eg, epidemiological surveillance) [13].

A full review of the AI field is beyond the scope of this review, and we refer the reader to some surveys (eg, [14]) and lectures $(\mathrm{eg},[15,16])$. However, we provide a compact overview of the AI-based techniques occurring most frequently in included studies in Multimedia Appendix 1.

\section{Research Problem}

AI has the ability to analyze big data sets through aggregating and sifting through mountains of health care data (including patient data) to generate insights that can enable predictive analysis. The quick ability to obtain these insights helps clinicians as well as other stakeholders in the health care ecosystem to make effective, safe, and timely decisions to better serve patients and public health policy. There has been a steady rise in the number of studies regarding the use of AI techniques to resolve or address the COVID-19 pandemic [13]. Much of the AI research effort during the COVID-19 pandemic has been scattered, and a need to explore and summarize how AI technologies are being used to resolve or address the many challenges relating to COVID-19 can help us plan on how to leverage AI technologies in the current or a future pandemic. Several reviews have been conducted on AI techniques used to address the COVID-19 pandemic [12,13,17-20]. However, much of the work has been in the form of literature reviews $[12,13,17-19]$ or systematic reviews focusing on one application of AI (eg, diagnosis and prognosis of COVID-19) [20]. Therefore, it is necessary to conduct a more systematic and comprehensive review that focuses on all applications of AI used amid the COVID-19 pandemic. Accordingly, this review aims to explore how AI technology is being used during the COVID-19 pandemic as reported in the literature. The results can be useful for health care professionals and policy makers considering leveraging AI to complement public health efforts in response to COVID-19.

\section{Methods}

To achieve the objective of this study while ensuring both replicable and transparent methods, we conducted a scoping review following the guidelines of PRISMA-ScR (Preferred Reporting Items for Systematic Reviews and Meta-Analyses Extension for Scoping Reviews) [21]. Methods used in this review are detailed in the following subsections.

\section{Search Strategy}

\section{Search Sources}

In this review, we performed search queries between April 10 and 12, 2020, on the following online databases: MEDLINE (via Ovid), EMBASE (via Ovid), PsycInfo (via Ovid), IEEE Xplore, ACM Digital Library, arXiv, medRxiv, bioRxiv, Scopus, and Google Scholar. In the case of Google Scholar and due to the volume of returned hits, only the first 100 results were considered, as we found that, beyond this, results quickly lose relevance and applicability. In addition to searching bibliographic databases, we screened the reference list of the included studies and relevant reviews to look for other relevant studies that could be added to this review (ie, backward reference list checking).

\section{Search Terms}

The search terms we used to identify relevant studies were specified from the available literature and by referring to subject matter experts. These terms were selected based on the target intervention (eg, AI, machine learning, and deep learning) and the target disease (eg, coronavirus, COVID-19, and 2019-nCoV). Details about the exact search strings used in this study are provided in Multimedia Appendix 2. 


\section{Study Eligibility Criteria}

In this review, we focused on any AI-based technology or approach used for any purpose related to the COVID-19 pandemic, such as diagnosis, epidemiological predictions, treatment and vaccine discovery, and prediction of patient outcomes. However, we excluded studies providing an overview or proposing a potential AI technique for COVID-19, or studies that were purely discussed from a research perspective.

We considered studies published in English between December 25, 2019, and April 12, 2020, such as peer-reviewed articles, theses, dissertations, conference proceedings, and preprints, while excluding other publications such as reviews, conference abstracts, proposals, editorials, and commentaries. We did not enforce any restrictions on the country of publication, study design, comparator, and outcomes.

\section{Study Selection}

Two reviewers, namely, authors AAA and MA, independently screened the titles and abstracts of the identified studies. They independently read the full text of studies that passed the title and abstract screening. We then investigated any disagreement between AAA and MA and resolved them through discussion and consensus. We calculated Cohen kappa [22] to measure the reviewer's agreement and found it to be 0.83 for the title and abstract screening and 0.94 for the full-text reading, indicating a very good agreement [23]. Multimedia Appendix 3 shows a matrix of interrater agreement in each step.

\section{Data Extraction}

Multimedia Appendix 4 shows a purpose-built data extraction form, which was pilot-tested using 7 relevant studies to accurately extract data. The two reviewers (AAA and MA) independently extracted data related to characteristics of the included studies, AI techniques, and data sets used for the development and validation of AI models. Like the study selection process, any disagreement between the reviewers was resolved through consensus. We calculated Cohen kappa [22] and found it to be 0.88 , meaning a very good agreement [23].

\section{Data Synthesis}

After extracting the data from the identified studies, we used a narrative approach to synthesize it. Specifically, we classified and described AI techniques used in the included studies in terms of their purposes (eg, diagnosis and drug and vaccine development), AI area or branch (eg, traditional machine learning and deep learning), AI models and algorithms (eg, decision tree, random forest, and naive Bayes), and platform (ie, computer and mobile). Further, we described the data sets used for development and validation of AI models in terms of data sources (eg, public databases and clinical settings); type of data (eg, radiology images, biological data, and laboratory data); size of the data set; type of validation; and proportion of training, validation, and test data sets. We used Microsoft Excel (Microsoft Corporation) to manage data synthesis.

\section{Results}

\section{Search Results}

We retrieved 435 studies through searching the identified bibliographic databases (Figure 1). Of those studies, we removed 53 duplicates; we then screened the titles and abstracts of the remaining 382 studies. The screening process led to the exclusion of 234 studies for reasons detailed in Figure 1. After reading the full texts of the remaining 148 studies, we excluded 73 studies, as they did not meet all eligibility criteria. Thus, we included the remaining 75 studies. We identified 7 additional studies by checking reference lists of the included studies and relevant literature reviews. Overall, 82 studies were included in this review. 
Figure 1. Flowchart of the study selection process.

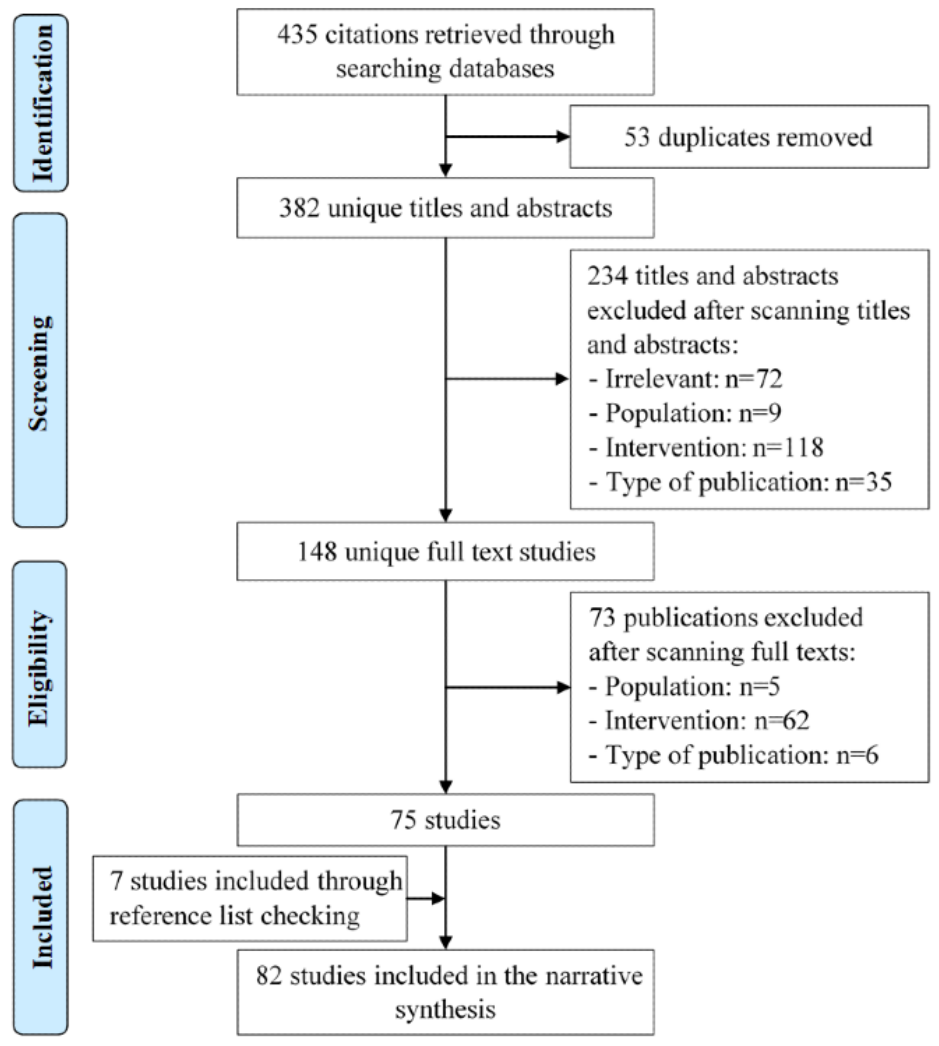

\section{Characteristics of the Included Studies}

Among the included studies, 72 were preprints and 10 were published articles in peer-reviewed journals (Table 1 and Figure $2)$. About two-thirds $(n=53)$ of the included studies were submitted in March 2020, and the remaining studies were published in February and April 2020. However, no studies were published during the first 2 months of the COVID-19 outbreak. The included studies were conducted in 19 countries; however, half of the studies $(n=41)$ were published in China. Multimedia Appendix 5 shows the characteristics of each included study. 
Table 1. Characteristics of the included studies.

\begin{tabular}{|c|c|}
\hline Characteristics & Studies $(\mathrm{N}=82), \mathrm{n}$ \\
\hline \multicolumn{2}{|l|}{ Paper status } \\
\hline Preprint & 72 \\
\hline Published & 10 \\
\hline \multicolumn{2}{|c|}{ Submission month } \\
\hline February & 13 \\
\hline March & 53 \\
\hline April & 16 \\
\hline \multicolumn{2}{|c|}{ Country of publication } \\
\hline China & 41 \\
\hline US & 9 \\
\hline India & 6 \\
\hline Turkey & 5 \\
\hline Canada & 4 \\
\hline UK & 3 \\
\hline Bangladesh & 2 \\
\hline Austria & 1 \\
\hline Egypt & 1 \\
\hline Greece & 1 \\
\hline Hong Kong & 1 \\
\hline Hungary & 1 \\
\hline Japan & 1 \\
\hline Korea & 1 \\
\hline Netherlands & 1 \\
\hline Pakistan & 1 \\
\hline Qatar & 1 \\
\hline Sudan & 1 \\
\hline Switzerland & 1 \\
\hline
\end{tabular}


Figure 2. Publications by months and country.

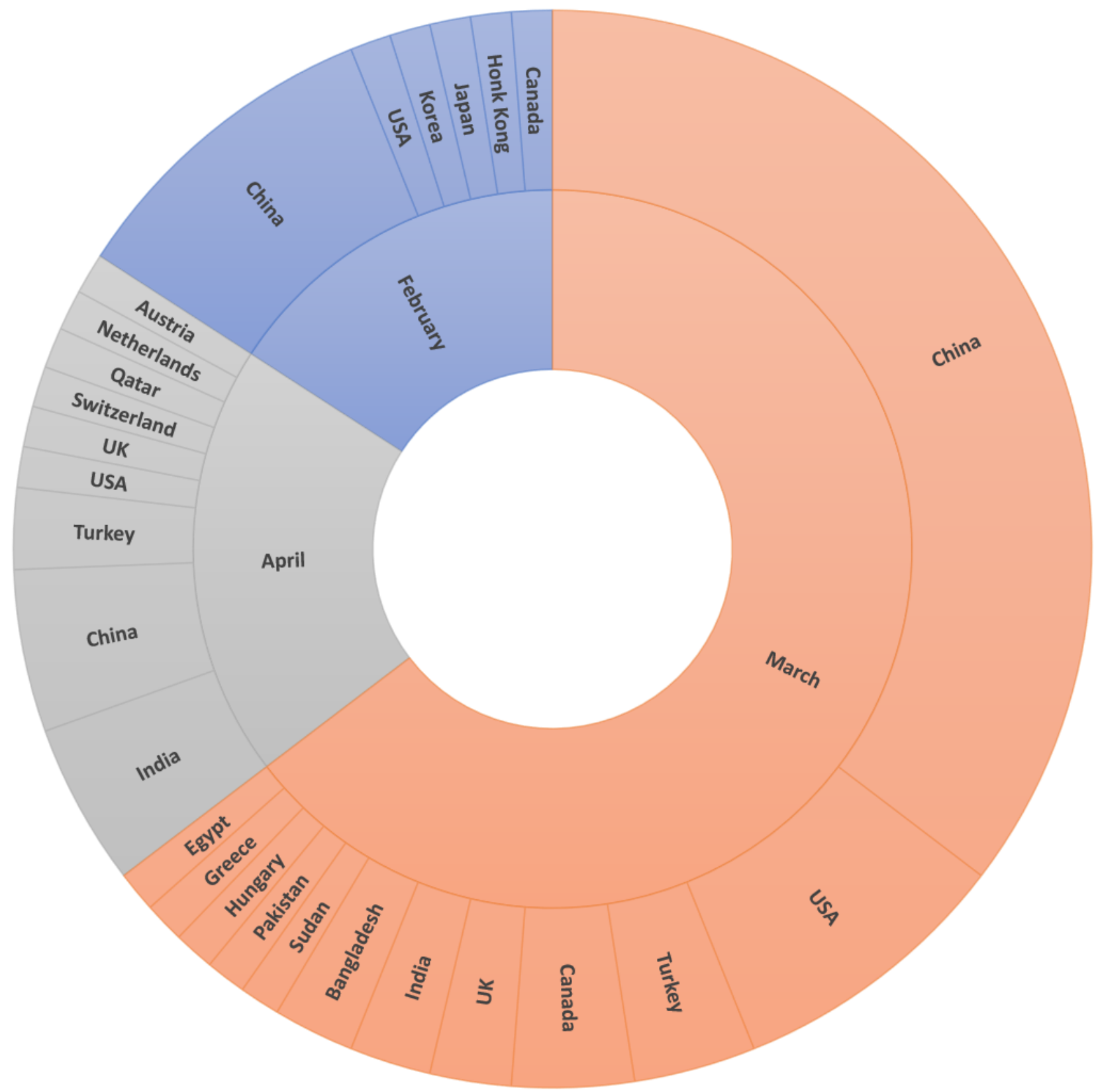

\section{AI-Based Techniques Used for COVID-19}

\section{Purposes or Uses of AI Against COVID-19}

As shown in Table 2, AI techniques have been used for five purposes amid the pandemic. In 31 studies [24-54], AI was used for diagnosing COVID-19 cases or identifying suspected COVID-19 cases based on various indicators, including computed tomography (CT) images [24-38], x-ray images [39-50], laboratory tests [51,52], genome sequences [53], and respiratory patterns [54]. 
Table 2. Purposes and uses of artificial intelligence against COVID-19.

\begin{tabular}{|c|c|}
\hline Purposes/uses & Studies $(\mathrm{N}=82), \mathrm{n}$ \\
\hline \multicolumn{2}{|l|}{ Diagnosis } \\
\hline $\mathrm{CT}^{\mathrm{a}}$ images & 15 \\
\hline $\mathrm{X}$-ray images & 12 \\
\hline Laboratory tests & 2 \\
\hline Genome sequence & 1 \\
\hline Respiratory patterns & 1 \\
\hline \multicolumn{2}{|l|}{ Treatment and vaccines } \\
\hline Drug discovery & 9 \\
\hline Vaccine discovery & 4 \\
\hline Protein structure & 4 \\
\hline Drug repurposing & 2 \\
\hline Treatment safety & 1 \\
\hline \multicolumn{2}{|l|}{ Epidemiology } \\
\hline Epidemic development & 14 \\
\hline Potential reservoirs & 3 \\
\hline \multicolumn{2}{|l|}{ Patient outcome } \\
\hline Severity & 6 \\
\hline Progression to severe & 4 \\
\hline Mortality risk & 2 \\
\hline Risk factors & 1 \\
\hline Hospital stay & 1 \\
\hline \multicolumn{2}{|l|}{ Infodemiology } \\
\hline Raising awareness & 1 \\
\hline
\end{tabular}

${ }^{\mathrm{a}} \mathrm{CT}$ : computed tomography.

In 20 studies [55-74], AI was also harnessed for treatment and vaccines for COVID-19. Specifically, 9 studies used AI for discovering drugs suitable for combating COVID-19 [55-63], and 2 studies used AI for repurposing commercially available drugs that could be used for treating COVID-19 [64,65]. There was 1 study that employed AI to predict the safety of using traditional Chinese medicine for COVID-19 [66]. In 4 studies [67-70], AI was used for discovering COVID-19 vaccines. Another 4 studies used AI for predicting the protein structure of SARS-CoV-2, thereby aiding researchers and pharmaceutical companies to discover drugs for COVID-19 [71-74].

There were 17 studies that used AI for epidemiological modeling tasks [75-91]. In particular, 14 of these studies employed AI for forecasting the epidemic development (eg, numbers of confirmed, recovered, death, suspected, asymptomatic, and critical cases, and lengths and ending time) [75-88], and 3 studies [89-91] used AI for predicting the potential hosts or reservoirs of SARS-CoV-2.

In 14 studies [33,92-104], AI was used for patient outcome-related tasks. In particular, 6 studies used AI for segmentation and quantification of infected regions in the lungs due to COVID-19, thereby assessing the severity of the disease [92-97]. AI was also used in 4 studies for identifying cases at high risk of progression to severe COVID-19 [33,98-100]. Furthermore, AI was also used for predicting mortality risk $[101,102]$, its associated factors [103], and the length of hospital stay in patients with COVID-19 [104].

AI has also been used for infodemiology [105]. Specifically, AI was used for raising awareness to use water, sanitation, and hygiene through combining authentic sources of information with daily news [105]. Multimedia Appendix 5 presents the purposes or uses of AI techniques in each included study.

\section{Features of AI-Based Techniques Used for COVID-19}

In 29 studies $[24,31,47, \quad 51,52,58, \quad 63,68,70$, $74,79,83-86,88,90,91,95-105]$, AI techniques used against COVID-19 were based on traditional machine learning models and algorithms (Table 3). The most commonly used machine learning models and algorithms were support vector machine (SVM) [24, 31, 47,58, 68, 70,79,91,98,101], random forest $[31,58,68,74,90,96,101,103,104], \quad$ decision tree $[52,58,68,74,79,97,99,101,102]$, and logistic regression [31,51,52,58,68,99-101,104]. 
Table 3. Features of AI-based techniques used for COVID-19.

\begin{tabular}{|c|c|}
\hline Features & Studies $(\mathrm{N}=82), \mathrm{n}$ \\
\hline \multicolumn{2}{|l|}{$\mathrm{AI}^{\mathrm{a}}$ branches $^{\mathrm{b}}$} \\
\hline Deep learning & 60 \\
\hline Machine learning & 29 \\
\hline Natural language processing & 3 \\
\hline \multicolumn{2}{|l|}{ AI models/ algorithms ${ }^{c}$} \\
\hline Convolutional neural network & 37 \\
\hline Support vector machine & 10 \\
\hline Random forest & 9 \\
\hline Decision tree & 9 \\
\hline Logistic regression & 9 \\
\hline Recurrent neural network & 8 \\
\hline Artificial neural network (unspecified) & 6 \\
\hline Transfer learning & 4 \\
\hline Autoencoders & 4 \\
\hline Deep neural network & 3 \\
\hline K-nearest neighbors & 3 \\
\hline Least absolute shrinkage and selection operator & 3 \\
\hline Polynomial neural network & 3 \\
\hline Multilayer perceptron & 2 \\
\hline Advance deep Q-learning network & 2 \\
\hline AdaBoost & 1 \\
\hline Auto-regressive integrated moving average model & 1 \\
\hline Bayesian analysis & 1 \\
\hline Bidirectional encoder representations from transformers & 1 \\
\hline Continuous bag of words & 1 \\
\hline Eureqa modeling & 1 \\
\hline Genetic algorithm & 1 \\
\hline Generative adversarial network & 1 \\
\hline Generalized logistic growth model & 1 \\
\hline Holistic agent-based model & 1 \\
\hline Linear discriminant analysis & 1 \\
\hline Linear regression & 1 \\
\hline Language model & 1 \\
\hline Multi-task deep model & 1 \\
\hline Naive Bayes & 1 \\
\hline Porter stemming & 1 \\
\hline Reinforcement learning & 1 \\
\hline Skip-gram model & 1 \\
\hline Time series forecasting & 1 \\
\hline Universal-sentence-encoder-large & 1 \\
\hline Vector auto average & 1 \\
\hline
\end{tabular}




\begin{tabular}{ll}
\hline Features & Studies $(\mathrm{N}=82), \mathrm{n}$ \\
\hline Platforms & \\
Computer & 81 \\
Mobile & 1 \\
\hline
\end{tabular}

\begin{abstract}
${ }^{\mathrm{a}} \mathrm{AI}$ : artificial intelligence.
${ }^{\mathrm{c}}$ Numbers do not add up as several studies used more than one AI model or algorithm.

In 60 studies, AI techniques used against COVID-19 were based on deep learning models and algorithms [25-50,53-57,59-67,69,71-73,75-82,87,89,92-95,98,101]. The most commonly used learning models and algorithms in the included studies were convolutional neural network (CNN) $[25-50,53,62,64,72,73,82,89,92-95]$ and recurrent neural network (RNN) [54,55,57,59,71,73,77,98].
\end{abstract}

${ }^{\mathrm{b}}$ Numbers do not add up as AI techniques in some studies were based on more than one AI branch.

In 2 studies [64,105], AI techniques used against COVID-19 were based on models related to natural language processing (NLP), such as the continuous bag of words model, skip-gram models, and porter stemming. Although AI techniques were implemented in mobile phones for 1 study [105], computers were the platform for AI techniques in the remaining studies. Multimedia Appendix 5 shows features of AI-based techniques used in each included study.

\section{Features of Data Sets Used for Development and Validation of AI Models}

As shown in Table 4, public resources (eg, National Center for Biotechnology Information [NCBI], GitHub, and Kaggle) were the most commonly used data source for development and validation of AI models [24,27, 29,36,39-50,53, 55-65,67-75,77, $80-85,87-89,91-93,103,105]$. Other data sources used by the included studies were as follows: clinical settings (eg, databases in hospitals and medical centers) [25-35,37,38,51,52,63,94-98,100, 102,104], government sources (eg, Chinese Center for Disease Control and Prevention) [53,76,78, 79, 84,86, 90, 99,101], literature (eg, previous studies and books) [36,40,42,61,66,101], news websites [101,105], and participants recruited by the study [54]. 
Table 4. Features of data sets used for development and validation of artificial intelligence models.

\begin{tabular}{|c|c|}
\hline Features & Studies (N=82), $\mathrm{n}$ \\
\hline \multicolumn{2}{|l|}{ Data sources $^{a}$} \\
\hline Public databases & 52 \\
\hline Clinical settings & 24 \\
\hline Government sources & 9 \\
\hline Literature & 6 \\
\hline News websites & 2 \\
\hline Participants & 1 \\
\hline \multicolumn{2}{|l|}{ Data types ${ }^{b}$} \\
\hline Radiology image & 35 \\
\hline Biological data & 23 \\
\hline Epidemiological data & 15 \\
\hline Clinical data & 11 \\
\hline Laboratory data & 8 \\
\hline Demographic data & 5 \\
\hline Guidelines & 1 \\
\hline News articles & 1 \\
\hline \multicolumn{2}{|l|}{ Data set size ${ }^{c}$} \\
\hline$<1000$ & 26 \\
\hline 1000-9999 & 16 \\
\hline$\geq 10,000$ & 8 \\
\hline \multicolumn{2}{|l|}{ Type of validation ${ }^{d, e}$} \\
\hline Train-test split & 25 \\
\hline K-fold cross-validation & 18 \\
\hline External validation & 11 \\
\hline \multicolumn{2}{|c|}{ Proportion of training set $(\%)^{f}$} \\
\hline$\leq 25$ & 3 \\
\hline $26-50$ & 2 \\
\hline $51-75$ & 16 \\
\hline$>75$ & 28 \\
\hline \multicolumn{2}{|c|}{ Proportion of validation set $(\%)^{\mathrm{g}}$} \\
\hline$\leq 25$ & 8 \\
\hline $26-50$ & 3 \\
\hline $51-75$ & 0 \\
\hline$>75$ & 0 \\
\hline \multicolumn{2}{|l|}{ Proportion of test set $(\%)^{h}$} \\
\hline$\leq 25$ & 35 \\
\hline $26-50$ & 10 \\
\hline $51-75$ & 3 \\
\hline$>75$ & 1 \\
\hline
\end{tabular}

${ }^{\mathrm{a}}$ Numbers do not add up as several studies collected their data from more than one data source. 
${ }^{b}$ Numbers do not add up as several studies collected more than one type of data.

${ }^{\mathrm{c}}$ Data set size was reported in 50 studies.

${ }^{\mathrm{d}}$ Type of validation was reported in 53 studies.

${ }^{\mathrm{e}}$ Numbers do not add up as 1 study used two different types of validation.

${ }^{f}$ Proportion of the training set was reported in 49 studies.

g Proportion of the validation set was reported in 11 studies.

${ }^{\mathrm{h}}$ Proportion of the test set was reported in 49 studies.

The types of data collected from these data sources were as follows: radiology images (eg, CT and x-ray) [24-50,54,92-96,98,104], biological data (eg, protein and genome sequences) [53,55-65,67-74,89-91], epidemiological data (eg, number of infected and recovered cases) [75-85,87,88,97,102], clinical data (eg, signs, symptoms, physician notes, and patients' history) [25,51,52,66,97-103], laboratory data (blood and polymerase chain reaction test results) $[25,51,52,86,97,98,100,102]$, demographic data (eg, age, gender, and ethnicity) [52,99-102], guidelines [105], and news articles [105].

The data set size was reported by 50 studies, ranging from 31 to $3,000,000$. The data set size was less than 1000 samples in half of these studies [24,27,32,34, 36,37,39, 41,44,45, $47,51-53,69,86,92,94-98,100,102-104]$, and only 8 studies reported a size of 10,000 samples or more $[25,26,54,59,61,87,99,101]$.

Validation of models was reported in 53 studies. Three types of validation were used in the included studies: train-test split $[25,29,30,34-39,41,43,44,47-50, \quad 59,75,87,88$, $93,94,99,100,103]$, K-fold cross-validation [24,31,40, 42,45,46, $52,53,58,66,68,90-92,96,98,101,104]$, and external validation $[26,27,29,32,33,38,51,54,82,95,102]$.

The training set proportion of the total data set was reported in 49 studies. The proportion of the training set ranged from $\leq 25 \%$ in 3 studies [25,27,28], 26\%-50\% in 2 studies [26,95], 51\%-75\% in 16 studies $[32,33,35,36, \quad 38, \quad 39,47, \quad 51$, $59,75,87,88,96,100,102,103]$, and $>75 \%$ in 28 studies $[24,29-31,34,37,40-46,48,50, \quad 52-54,58, \quad 66,90-92$, $94,98,99,101,104]$. The mean of the proportions of the training set in the 49 studies was $72.7 \%$.

The validation set proportion of the total data set was reported in 11 studies; it ranged from $\leq 25 \%$ in 8 studies $[26,28,35,36,38$, $47,48,53]$ and $26 \%-50 \%$ in 3 studies $[25,100,102]$. The mean of the validation set proportions in the 11 studies was $18.7 \%$.

The test set proportion of the total data set was reported in 49 studies, ranging from $\leq 25 \%$ in 35 studies [24,29-31,34,36,37,40-48,50,52-54,58,59,66,75,87,90-92,94,98-102,104], $26 \%-50 \%$ in 10 studies $[26,32,33,35,38,39,51,88,96,103]$, $51 \%-75 \%$ in 3 studies $[25,28,95]$, and $>75 \%$ in 1 study [27]. The mean of the test set proportions in the 49 studies was $22.9 \%$. Multimedia Appendix 6 presents features of the data sets used for development and validation of AI models in each included study.

\section{Discussion}

\section{Principal Results}

In this study, we conducted a scoping review of the use of AI against COVID-19. We found a lack of publications in December 2019 and January 2020. This is not surprising, given that SARS-CoV-2 was only identified on January 7 [106]; insufficient data was not available to back scientific publications, in particular internationally; and the contagiousness and aggressiveness of the virus were underestimated (first lockdown in China was January 23 [106]). Half of the studies in this report were published in China. Since SARS-CoV-2 originated in China and affected it the most during the first 3 months of the pandemic, it had the most data related to COVID-19. Considering lengthy publication processes and the vast number of COVID-19-related manuscript submissions, it is also not surprising that most of the included studies were preprints.

In the included studies, AI was used for five purposes: diagnosis, treatment and vaccine discovery, epidemiological modeling, patient outcome-related tasks, and infodemiology. None of the included studies used AI for other purposes such as contact tracing of the individuals, providing training to students and health care professionals, or robotics to deal with suspected and quarantined cases.

Most of the AI techniques used in the included studies were based on deep learning approaches such as CNN and RNN. All but 1 study used desktop machines, workstations, and clusters as opposed to mobile platforms. This can be explained by the computational demand in training AIs. Although all major mobile phone manufacturers equip their flagship models with AI coprocessors, these coprocessors accelerate inference, a computationally much lighter task. In addition, federated learning [107] (a machine learning privacy-preserving technique usually used in mobile phones) is still in its infancy and raises issues such as data sovereignty, scalability, and performance.

Data sources used in the included studies usually came from the public domain (eg, NCBI, GitHub, Kaggle) and proprietary databases (less common). Radiology images were the most commonly used type of data, followed by biological data. The number of samples was still comparably small (less than 1000 in half of the studies). The diversity and size of data indicate a lack of publicly available data despite COVID-19 cases having surpassed 5 million at the time of writing. We, therefore, second Wynants et al [20] call "for immediate sharing of the individual participant data from COVID-19 studies worldwide."

\section{Practical and Research Implications}

Although this review explores the use of AI against COVID-19, some applications could prove useful far beyond this pandemic. 
For instance, Kiwibot designs autonomous medical delivery robots to minimize interpersonal contact [108]. Whiteboard Coordinator developed a high precision thermal screening device eliminating individual measurements, leading to higher throughput and larger social distances [109]. Although mobile phones are not yet the AI platform of choice, the first apps to track interpersonal contact using mobile phones have been published to prioritize COVID-19 testing [110]. Finally, whereas a real-time reverse transcription polymerase chain reaction test takes around 25 minutes and requires stocks of chemical reagents, AI can inspect chest CTs to provide preliminary diagnoses in seconds. We believe that increasing social distance and providing fully autonomous checkups will be the most valuable use of AI beyond the current pandemic.

In the past, fundamental AI research was focused mainly on faster (or even feasible) training. We believe that, in the future, this must be complemented with public education. AI mistrust, because of our still lacking understanding of how AI works at the deepest level, further raises ethical questions that need to be answered before AI will be uniformly accepted. We also found that AI features and results were reported in an inconsistent manner, potentially fueling AI mistrust and making a direct comparison between studies difficult. Of the 82 studies, we found that only $64.6 \%(n=53)$ of the studies included in this review disclosed the type of validation, $61 \%(n=50)$ mentioned the data size, and more than $7 \%(n=6)$ did not even specify the type of AI used. It is therefore important that we as a community develop a standardized reporting protocol to slow down the barrage of poorly conducted COVID-19 studies that threaten to overwhelm serious scientists (1916 related papers were retrieved before April 5, 2020 by Wynants et al [20]), strengthen properly conducted studies, and improve reproducibility.

We found that, explicably, the landscape of studies is still dominated by Chinese institutions, which bears the potential for cultural, technological, and geospatial biases. However, we see a recent move toward a more balanced landscape (see Figure 2). Although we identified more than 100 models developed in the included studies, we did not assess their quality as it was out of this review's scope. Therefore, further reviews are needed to assess the quality of AI models used in the fight against COVID-19.

Given the current "infodemic" [13], we find it surprising that NLP is not used more often. We see AI-based analysis of effective advertisement for nonpharmaceutical interventions as one research opportunity to answer questions like what manner of speech results in maximum public acceptance.

\section{Strengths and Limitations}

\section{Strengths}

Given that this review includes all AI techniques used for the COVID-19 pandemic regardless of their characteristics, study design, study setting, and country of publication, it may be considered the most comprehensive review in this research area. This helps readers to speculate how AI is being leveraged amid the COVID-19 pandemic. In comparison with similar reviews $[12,13,17-20]$, our review is the only one that describes and summarizes features of the identified AI techniques and data sets used for their development and validation. Furthermore, unlike previous reviews [12,13,17-19], this review follows the full scientific rigor of PRISMA-ScR [21].

In contrast to other reviews, we searched the most commonly used databases in health and information technology fields to identify as many relevant studies as possible. Thus, the number of studies included in this review was much higher than in other reviews [12,13,17-20]. Additionally, we strove to retrieve gray literature and minimized the risk of publication bias by searching Google Scholar and conducting backward reference list checking. Furthermore, we minimized selection bias by having two independent reviewers conducting study selection and data extraction, with a very high agreement in both processes.

\section{Limitations}

Given that our review excludes proposals of AI techniques, it is likely that we missed other applications of AI for COVID-19. This review, therefore, might not identify all potential uses of AI for the current pandemic. Owing to practical constraints, the search was restricted to English studies. Therefore, we probably missed several studies written in other languages, especially Chinese. The search query did not include terms related to specific types of models or algorithms such as CNN, RNN, and SVM. Thus, it is likely that we missed some studies that used such terms in their title and abstract instead of the terms that we used (ie, AI, machine learning, and deep learning). The findings of this review are mostly based on preprints, which are more likely to have inaccurate or missing information. Therefore, the accuracy of the information in the included studies may affect the accuracy of our findings.

\section{Conclusions}

In this study, we provide a scoping review of 82 studies on AI against COVID-19. Given that many of the proposed methods are not yet clinically accepted, we remark that the most rewarding research will be on methods promising value beyond COVID-19. We believe that mobile phones offer unexploited potential, but more research in the direction of energy-efficient and federated learning is needed. We also believe that the use of NLP to assess effective communication of nonpharmaceutical interventions is a largely unexplored research direction, especially since data driving this research is available in the public domain, unlike much of the data produced by clinical studies. For AI to gain broad acceptance, standardized reporting protocols to be followed by studies on AI are needed. Likewise, more research on AI ethics and explainable $\mathrm{AI}$ is needed, paired with public education initiatives.

\section{Conflicts of Interest}

None declared. 


\section{Multimedia Appendix 1}

Overview of artificial intelligence-based techniques.

[DOCX File, 14 KB-Multimedia Appendix 1]

\section{Multimedia Appendix 2}

Search strategy.

[DOCX File, 19 KB-Multimedia Appendix 2]

\section{Multimedia Appendix 3}

Interrater agreement matrices for study selection steps.

[DOCX File, 13 KB-Multimedia Appendix 3]

\section{Multimedia Appendix 4}

Data extraction form.

[DOCX File, 17 KB-Multimedia Appendix 4]

\section{Multimedia Appendix 5}

Characteristics of the included studies and features of artificial intelligence techniques used for COVID-19.

[DOCX File, 26 KB-Multimedia Appendix 5]

\section{Multimedia Appendix 6}

Features of data sets used for the development and validation of artificial intelligence models.

[DOCX File, 25 KB-Multimedia Appendix 6]

\section{References}

1. Kong W, Li Y, Peng M, Kong D, Yang X, Wang L, et al. SARS-CoV-2 detection in patients with influenza-like illness. Nat Microbiol 2020 May;5(5):675-678. [doi: 10.1038/s41564-020-0713-1] [Medline: 32265517]

2. Coronavirus disease (COVID-19): situation report- 121. World Health Organization. 2020. URL: https://www.who.int/ docs/default-source/coronaviruse/situation-reports/20200520-covid-19-sitrep-121.pdf [accessed 2020-04-19]

3. Chen Y, Li L. SARS-CoV-2: virus dynamics and host response. Lancet Infect Dis 2020 May;20(5):515-516 [FREE Full text] [doi: $\underline{10.1016 / S 1473-3099(20) 30235-8]}$ [Medline: $\underline{32213336]}$

4. Del Rio C, Malani PN. COVID-19-new insights on a rapidly changing epidemic. JAMA 2020 Apr 14;323(14):1339-1340. [doi: 10.1001/jama.2020.3072] [Medline: 32108857]

5. Fontanarosa PB, Bauchner H. COVID-19-looking beyond tomorrow for health care and society. JAMA 2020 May 19;323(19):1907-1908. [doi: 10.1001/jama.2020.6582] [Medline: 32301955]

6. Yang P, Wang X. COVID-19: a new challenge for human beings. Cell Mol Immunol 2020 May; 17(5):555-557 [FREE Full text] [doi: 10.1038/s41423-020-0407-x] [Medline: 32235915]

7. Ting DSW, Carin L, Dzau V, Wong TY. Digital technology and COVID-19. Nat Med 2020 Apr;26(4):459-461 [FREE Full text] [doi: 10.1038/s41591-020-0824-5] [Medline: 32284618]

8. Contreras I, Vehi J. Artificial intelligence for diabetes management and decision support: literature review. J Med Internet Res 2018 May 30;20(5):e10775 [FREE Full text] [doi: 10.2196/10775] [Medline: 29848472]

9. Shaw J, Rudzicz F, Jamieson T, Goldfarb A. Artificial intelligence and the implementation challenge. J Med Internet Res 2019 Jul 10;21(7):e13659 [FREE Full text] [doi: 10.2196/13659] [Medline: 31293245]

10. van Hartskamp M, Consoli S, Verhaegh W, Petkovic M, van de Stolpe A. Artificial intelligence in clinical health care applications: viewpoint. Interact J Med Res 2019 Apr 05;8(2):e12100 [FREE Full text] [doi: 10.2196/12100] [Medline: 30950806]

11. Russell S, Norvig P. Artificial Intelligence: A Modern Approach, third edition. Upper Saddle River, NJ: Prentice Hall; 2010.

12. Alimadadi A, Aryal S, Manandhar I, Munroe PB, Joe B, Cheng X. Artificial intelligence and machine learning to fight COVID-19. Physiol Genomics 2020 Apr 01;52(4):200-202 [FREE Full text] [doi: 10.1152/physiolgenomics.00029.2020] [Medline: $\underline{32216577]}$

13. Bullock J, Luccioni A, Hoffman Pham K, Sin Nga Lam C, Luengo-Oroz M. Mapping the landscape of artificial intelligence applications against COVID-19. arXiv. Preprint posted online March 25, 2020 . [doi: 10.1613/jair.1.12162]

14. Mondal B. Artificial intelligence: state of the art. In: Balas VE, Kumar R, Srivastava R, editors. Recent Trends and Advances in Artificial Intelligence and Internet of Things. New York, NY: Springer; 2019:389-425. 
15. Fridman L. Deep learning state of the art (2020). YouTube. 2020. URL: https://www.youtube.com/ watch?v=0VH1Lim8gL8\&list=PLrAXtmErZgOeiKm4sgNOknGvNjby9efdf [accessed 2020-07-23]

16. ACMSIGGRAPH. Deep learning: a crash course. YouTube. 2018. URL: https://www.youtube.com/watch?v=r0Ogt-q956I [accessed 2020-07-23]

17. Santosh KC. AI-driven tools for coronavirus outbreak: need of active learning and cross-population train/test models on multitudinal/multimodal data. J Med Syst 2020 Mar 18;44(5):93 [FREE Full text] [doi: 10.1007/s10916-020-01562-1] [Medline: 32189081]

18. Shi F, Wang J, Shi J, Wu Z, Wang Q, Tang Z, et al. Review of artificial intelligence techniques in imaging data acquisition, segmentation and diagnosis for COVID-19. IEEE Rev Biomed Eng 2020 Apr 16;PP:1. [doi: 10.1109/RBME.2020.2987975] [Medline: 32305937]

19. Vaishya R, Javaid M, Khan I, Haleem A. Artificial intelligence (AI) applications for COVID-19 pandemic. Diabetes Metab Syndr 2020;14(4):337-339 [FREE Full text] [doi: 10.1016/j.dsx.2020.04.012] [Medline: 32305024]

20. Wynants L, Van Calster B, Bonten MMJ, Collins GS, Debray TPA, De Vos M, et al. Systematic review and critical appraisal of prediction models for diagnosis and prognosis of COVID-19 infection. medRxiv. Preprint posted online April 5, 2020 . [doi: $\underline{10.1101 / 2020.03 .24 .20041020]}$

21. Tricco AC, Lillie E, Zarin W, O'Brien KK, Colquhoun H, Levac D, et al. PRISMA Extension for Scoping Reviews (PRISMA-ScR): checklist and explanation. Ann Intern Med 2018 Oct 02;169(7):467-473 [FREE Full text] [doi: 10.7326/M18-0850] [Medline: 30178033]

22. Higgins J, Deeks J. Chapter 7: selecting studies and collecting data. In: Cochrane Handbook for Systematic Reviews of Interventions. Hoboken, NJ: John Wiley \& Sons; 2008.

23. Altman DG. Practical Statistics for Medical Research. Boca Raton, FL: CRC Press; 1990.

24. Barstugan M, Ozkaya U, Ozturk S. Coronavirus (COVID-19) classification using CT images by machine learning methods. arXiv. Preprint posted online March 20, 2020 .

25. Chen J, Wu L, Zhang J, Zhang L, Gong D, Zhao Y, et al. Deep learning-based model for detecting 2019 novel coronavirus pneumonia on high-resolution computed tomography: a prospective study. medXriv. Preprint posted online March 1, 2020 . [doi: $10.1101 / 2020.02 .25 .20021568]$

26. Fu M, Yi SL, Zeng Y, Ye F, Li Y, Dong X, et al. Deep learning-based recognizing COVID-19 and other common infectious diseases of the lung by chest CT scan images. medRxiv. Preprint posted online March 30, 2020 . [doi:

$\underline{10.1101 / 2020.03 .28 .20046045]}$

27. Gozes O, Frid-Adar M, Greenspan H, Browning PD, Zhang H, Ji W, et al. arXiv. Preprint posted online March 10, 2020 .

28. Jin C, Chen W, Cao Y, Xu Z, Tan Z, Zhang X, et al. Development and evaluation of an AI system for COVID-19 diagnosis. medRxiv. Preprint posted online June 2, 2020 . [doi: 10.1101/2020.03.20.20039834]

29. Jin S, Wang B, Xu H, Luo C, Wei L, Zhao W, et al. AI-assisted CT imaging analysis for COVID-19 screening: building and deploying a medical AI system in four weeks. medRxiv. Preprint posted online March 23, 2020 . [doi:

10.1101/2020.03.19.20039354]

30. Li L, Qin L, Xu Z, Yin Y, Wang X, Kong B, et al. Using artificial intelligence to detect COVID-19 and community-acquired pneumonia based on pulmonary CT: evaluation of the diagnostic accuracy. Radiology 2020 Aug;296(2):E65-E71 [FREE Full text] [doi: 10.1148/radiol.2020200905] [Medline: $\underline{\text { 32191588] }}$

31. Shi F, Xia L, Shan F, Wu D, Wei Y, Yuan H, et al. Large-scale screening of COVID-19 from community acquired pneumonia using infection size-aware classification. arXiv. Preprint posted online March 22, 2020 .

32. Wang S, Kang B, Ma J, Zeng X, Xiao M, Guo J, et al. A deep learning algorithm using CT images to screen for corona virus disease (COVID-19). medRxiv. Preprint posted online April 24, 2020 . [doi: 10.1101/2020.02.14.20023028]

33. Wang S, Zha Y, Li W, Wu Q, Li X, Niu M, et al. A fully automatic deep learning system for COVID-19 diagnostic and prognostic analysis. medRxiv. Preprint posted online March 26, 2020 . [doi: 10.1101/2020.03.24.20042317]

34. Xu X, Jiang X, Ma C, Du P, Li X, Lv S, et al. Deep learning system to screen coronavirus disease 2019 pneumonia. arXiv. Preprint posted online February 21, 2020 .

35. Ying S, Zheng S, Li L, Zhang X, Zhang X, Huang Z, et al. Deep learning enables accurate diagnosis of novel coronavirus (COVID-19) with CT images. medRxiv. Preprint posted online February 25, 2020 . [doi: 10.1101/2020.02.23.20026930]

36. Yang X, He X, Zhao J, Zhang Y, Zhang S, Xie P. COVID-CT-Dataset: a CT scan dataset about COVID-19. arXiv. Preprint posted online March 30, 2020.

37. Zheng C, Deng X, Fu Q, Zhou Q, Feng J, Ma H, et al. Deep learning-based detection for COVID-19 from chest CT using weak label. medRxiv. Preprint posted online March 26, 2020 . [doi: 10.1101/2020.03.12.20027185]

38. Zhou M, Chen Y, Yang D, Xu Y, Yao W, Huang J, et al. Improved deep learning model for differentiating novel coronavirus pneumonia and influenza pneumonia. medRxiv. Preprint posted online March 30, 2020 . [doi: 10.1101/2020.03.24.20043117]

39. Abbas A, Abdelsamea M, Gaber M. Classification of COVID-19 in chest X-ray images using DeTraC deep convolutional neural network. medRxiv. Preprint posted online May 18, 2020 . [doi: 10.1101/2020.03.30.20047456]

40. Apostolopoulos I, Mpesiana TA. Covid-19: automatic detection from x-ray images utilizing transfer learning with convolutional neural networks. Phys Eng Sci Med 2020 Jun;43(2):635-640 [FREE Full text] [doi:

10.1007/s13246-020-00865-4] [Medline: 32524445] 
41. Bukhari SUK, Bukhari SSK, Syed A, Shah SSH. The diagnostic evaluation of convolutional neural network (CNN) for the assessment of chest X-ray of patients infected with COVID-19. medRxiv. Preprint posted online March 31, 2020 . [doi: 10.1101/2020.03.26.20044610]

42. Chowdhury MEH, Rahman T, Khandakar A, Mazhar R, Kadir MA, Mahbub ZB, et al. Can AI help in screening Viral and COVID-19 pneumonia? arXiv. Preprint posted online March 29, 2020 .

43. Ghoshal B, Tucker A. Estimating uncertainty and interpretability in deep learning for coronavirus (COVID-19) detection. arXiv. Preprint posted online March 22, 2020 .

44. Hemdan EED, Shouman MA, Karar ME. COVIDX-Net: a framework of deep learning classifiers to diagnose COVID-19 in X-ray images. arXiv. Preprint posted online March 24, 2020.

45. Narin A, Kaya C, Pamuk Z. Automatic detection of coronavirus disease (COVID-19) using X-ray images and deep convolutional neural networks. arXiv. Preprint posted online March 24, 2020 .

46. Ozturk T, Talo M, Yildirim EA, Baloglu UB, Yildirim O, Rajendra Acharya U. Automated detection of COVID-19 cases using deep neural networks with X-ray images. Comput Biol Med 2020 Jun;121:103792 [FREE Full text] [doi: 10.1016/j.compbiomed.2020.103792] [Medline: 32568675]

47. Sethy PK, Behera SK. Detection of coronavirus disease (COVID-19) based on deep features. Preprints. Preprint posted online March 19, 2020 . [doi: 10.20944/preprints202003.0300.v1]

48. Ucar F, Korkmaz D. COVIDiagnosis-Net: deep Bayes-SqueezeNet based diagnosis of the coronavirus disease 2019 (COVID-19) from X-ray images. Med Hypotheses 2020 Jul;140:109761 [FREE Full text] [doi: 10.1016/j.mehy.2020.109761] [Medline: 32344309]

49. Wang L, Lin ZQ, Wong A. COVID-Net: a tailored deep convolutional neural network design for detection of COVID-19 cases from chest X-ray images. Sci Rep 2020 Nov 11;10(1):19549. [doi: 10.1038/s41598-020-76550-z] [Medline: 33177550 ]

50. Zhang J, Xie Y, Li Y, Shen C, Xia Y. COVID-19 screening on chest X-ray images using deep learning based anomaly detection. arXiv. Preprint posted online March 27, 2020 .

51. Meng Z, Wang M, Song H, Guo S, Zhou Y, Li W, et al. Development and utilization of an intelligent application for aiding COVID-19 diagnosis. medRxiv. Preprint posted online March 21, 2020 . [doi: 10.1101/2020.03.18.20035816]

52. Feng C, Huang Z, Wang L, Chen X, Zhai Y, Zhu F, et al. A novel triage tool of artificial intelligence assisted diagnosis aid system for suspected COVID-19 pneumonia in fever clinics. medRxiv. Preprint posted online March 20, 2020 . [doi: 10.1101/2020.03.19.20039099]

53. Lopez-Rincon A, Tonda A, Mendoza-Maldonado L, Claassen E, Garssen J, Kraneveld AD. Accurate identification of SARS-CoV-2 from viral genome sequences using deep learning. bioRxiv. Preprint posted online March 14, 2020 . [doi: 10.1101/2020.03.13.990242]

54. Wang Y, Hu M, Li Q, Zhang XP, Zhai G, Yao N. Abnormal respiratory patterns classifier may contribute to large-scale screening of people infected with COVID-19 in an accurate and unobtrusive manner. arXiv. Preprint posted online February 12,2020 .

55. Gao K, Nguyen DD, Wang R, Wei GW. Machine intelligence design of 2019-nCoV drugs. bioRxiv. Preprint posted online February 4, 2020 . [doi: 10.1101/2020.01.30.927889]

56. Ge Y, Tian T, Huang S, Wan F, Li J, Li S, et al. A data-driven drug repositioning framework discovered a potential therapeutic agent targeting COVID-19. bioRxiv. Preprint posted online March 12, 2020 . [doi: 10.1101/2020.03.11.986836]

57. Hofmarcher M, Mayr A, Rumetshofer E, Ruch P, Renz P, Schimunek J, et al. Large-scale ligand-based virtual screening for SARS-CoV-2 inhibitors using deep neural networks. SSRN J. Preprint posted online March 26, 2020 . [doi: 10.2139/ssrn.3561442]

58. Magar R, Yadav P, Farimani AB. Potential neutralizing antibodies discovered for novel corona virus using machine learning. arXiv. Preprint posted online March 18, 2020 . [doi: 10.1101/2020.03.14.992156]

59. Patankar S. Deep learning-based computational drug discovery to inhibit the RNA Dependent RNA Polymerase: application to SARS-CoV and COVID-19. OSF Preprints. Preprint posted online March 18, 2020 . [doi: 10.31219/osf.io/6kpbg]

60. Tang B, He F, Liu D, Fang M, Wu Z, Xu D. AI-aided design of novel targeted covalent inhibitors against SARS-CoV-2. bioRxiv. Preprint posted online March 8, 2020 . [doi: 10.1101/2020.03.03.972133]

61. Ton A, Gentile F, Hsing M, Ban F, Cherkasov A. Rapid identification of potential inhibitors of SARS-CoV-2 main protease by deep docking of 1.3 billion compounds. Mol Inform 2020 Aug;39(8):e2000028 [FREE Full text] [doi: 10.1002/minf.202000028] [Medline: $\underline{\text { 32162456] }}$

62. Zhang H, Saravanan KM, Yang Y, Hossain MD, Li J, Ren X, et al. Deep learning based drug screening for novel coronavirus 2019-nCov. Preprints. Preprint posted online February 5, 2020 . [doi: 10.20944/preprints202002.0061.v1]

63. Zhavoronkov A, Aladinskiy V, Zhebrak A, Zagribelnyy B, Terentiev V, Bezrukov DS, et al. Potential 2019-nCoV 3C-like protease inhibitors designed using generative deep learning approaches. ChemRxiv. Preprint posted online February 10, 2020 . [doi: 10.26434/chemrxiv.11829102]

64. Beck BR, Shin B, Choi Y, Park S, Kang K. Predicting commercially available antiviral drugs that may act on the novel coronavirus (2019-nCoV), Wuhan, China through a drug-target interaction deep learning model. bioRxiv. Preprint posted online February 2, 2020 . [doi: 10.1101/2020.01.31.929547] 
65. Hu F, Jiang J, Yin P. Prediction of potential commercially inhibitors against SARS-CoV-2 by multi-task deep model. arXiv. Preprint posted online March 2, 2020 .

66. Wang Z, Li L, Yan J, Yao Y. Evaluating the traditional Chinese medicine (TCM) officially recommended in China for COVID-19 using ontology-based side-effect prediction framework (OSPF) and deep learning. Preprints. Preprint posted online February 17, 2020 . [doi: 10.20944/preprints202002.0230.v1]

67. Abdelmageed MI, Abdelmoneim AH, Mustafa MI, Elfadol NM, Murshed NS, Shantier SW, et al. Design of multi epitope-based peptide vaccine against E protein of human 2019-nCoV: an immunoinformatics approach. bioRxiv. Preprint posted online February 11, 2020 . [doi: 10.1101/2020.02.04.934232]

68. Ong E, Wong MU, Huffman A, He Y. COVID-19 coronavirus vaccine design using reverse vaccinology and machine learning. bioRxiv. Preprint posted online March 21, 2020 . [doi: 10.1101/2020.03.20.000141]

69. Rahman MS, Hoque MN, Islam MR, Akter S, Rubayet-Ul-Alam ASM, Siddique MA, et al. Epitope-based chimeric peptide vaccine design against S, M and E proteins of SARS-CoV-2 etiologic agent of global pandemic COVID-19: an in silico approach. bioRxiv. Preprint posted online March 31, 2020 . [doi: 10.1101/2020.03.30.015164]

70. Sarkar B, Ullah MA, Johora FT, Taniya MA, Araf Y. The essential facts of Wuhan novel coronavirus outbreak in China and epitope-based vaccine designing against 2019-nCoV. bioRxiv. Preprint posted online February 11, 2020 . [doi: 10.1101/2020.02.05.935072]

71. Fast E, Altman RB, Chen B. Potential T-cell and B-cell epitopes of 2019-nCoV. bioRxiv. Preprint posted online February 21, 2020 . [doi: 10.1101/2020.02.19.955484]

72. Heo L, Feig M. Modeling of severe acute respiratory syndrome coronavirus 2 (SARS-CoV-2) proteins by machine learning and physics-based refinement. bioRxiv. Preprint posted online March 28, 2020 . [doi: 10.1101/2020.03.25.008904]

73. Qiao R, Tran NH, Shan B, Ghodsi A, Li M. Personalized workflow to identify optimal T-cell epitopes for peptide-based vaccines against COVID-19. arXiv. Preprint posted online March 24, 2020 .

74. Demirci MDS, Adan A. Computational analysis of microRNA-mediated interactions in SARS-CoV-2 infection. bioRxiv. Preprint posted online March 17, 2020 . [doi: 10.1101/2020.03.15.992438]

75. Al-Qaness MAA, Ewees AA, Fan H, Abd El Aziz M. Optimization method for forecasting confirmed cases of COVID-19 in China. J Clin Med 2020 Mar 02;9(3):674 [FREE Full text] [doi: 10.3390/jcm9030674] [Medline: $\underline{\text { 32131537] }}$

76. Dandekar R, Barbastathis G. Neural network aided quarantine control model estimation of COVID spread in Wuhan, China. arXiv. Preprint posted online March 18, 2020 .

77. Dutta S, Bandyopadhyay SK. Machine learning approach for confirmation of COVID-19 cases: positive, negative, death and release. medRxiv. Preprint posted online March 30, 2020 . [doi: 10.1101/2020.03.25.20043505]

78. Fong SJ, Li G, Dey N, Crespo RG, Herrera-Viedma E. Composite Monte Carlo decision making under high uncertainty of novel coronavirus epidemic using hybridized deep learning and fuzzy rule induction. arXiv. Preprint posted online March 22,2020 .

79. Fong SJ, Li G, Dey N, Crespo RG, Herrera-Viedma E. Finding an accurate early forecasting model from small dataset: a case of 2019-nCoV novel coronavirus outbreak. arXiv. Preprint posted online March 24, 2020 .

80. Hu Z, Ge Q, Li S, Boerwincle E, Jin L, Xiong M. Forecasting and evaluating intervention of covid-19 in the world. arXiv. Preprint posted online March 22, 2020 .

81. Hu Z, Ge Q, Li S, Jin L, Xiong M. Artificial intelligence forecasting of covid-19 in China. arXiv. Preprint posted online February 17, 2020 .

82. Huang CJ, Chen YH, Ma Y, Kuo PH. Multiple-input deep convolutional neural network model for COVID-19 forecasting in China. medRxiv. Preprint posted online March 27, 2020 . [doi: 10.1101/2020.03.23.20041608]

83. Kumar P, Kalita H, Patairiya S, Sharma YD, Nanda C, Rani M, et al. Forecasting the dynamics of COVID-19 pandemic in top 15 countries in April 2020: ARIMA model with machine learning approach. medRxiv. Preprint posted online April 5, 2020 . [doi: 10.1101/2020.03.30.20046227]

84. Li M, Zhang Z, Jiang S, Liu Q, Chen C, Zhang Y, et al. Predicting the epidemic trend of COVID-19 in China and across the world using the machine learning approach. medRxiv. Preprint posted online April 20, 2020 . [doi: $10.1101 / 2020.03 .18 .20038117]$

85. Marini M, Chokani N, Abhari RS. COVID-19 epidemic in Switzerland: growth prediction and containment strategy using artificial intelligence and big data. medRxiv. Preprint posted online April 7, 2020 . [doi: 10.1101/2020.03.30.20047472]

86. Mizumoto K, Kagaya K, Zarebski A, Chowell G. Estimating the asymptomatic proportion of coronavirus disease 2019 (COVID-19) cases on board the Diamond Princess cruise ship, Yokohama, Japan, 2020. Euro Surveill 2020 Mar;25(10):2000180 [FREE Full text] [doi: 10.2807/1560-7917.ES.2020.25.10.2000180] [Medline: $\underline{\text { 32183930] }}$

87. Pirouz B, Shaffiee Haghshenas S, Shaffiee Haghshenas S, Piro P. Investigating a serious challenge in the sustainable development process: analysis of confirmed cases of COVID-19 (new type of coronavirus) through a binary classification using artificial intelligence and regression analysis. Sustainability 2020 Mar 20;12(6):2427. [doi: 10.3390/su12062427]

88. Tiwari S, Kumar S, Guleria K. Outbreak trends of coronavirus disease-2019 in India: a prediction. Disaster Med Public Health Prep 2020 Apr 22:1-6 [FREE Full text] [doi: 10.1017/dmp.2020.115] [Medline: 32317044]

89. Guo Q, Li M, Wang C, Wang P, Fang Z, Tan J, et al. Host and infectivity prediction of Wuhan 2019 novel coronavirus using deep learning algorithm. bioRxiv. Preprint posted online January 24, 2020 . 
90. Qiang X, Xu P, Fang G, Liu W, Kou Z. Using the spike protein feature to predict infection risk and monitor the evolutionary dynamic of coronavirus. Infect Dis Poverty 2020 Mar 25;9(1):33 [FREE Full text] [doi: 10.1186/s40249-020-00649-8] [Medline: $\underline{32209118]}$

91. Randhawa GS, Soltysiak MPM, El Roz H, de Souza CPE, Hill KA, Kari L. Machine learning using intrinsic genomic signatures for rapid classification of novel pathogens: COVID-19 case study. bioRxiv. Preprint posted online February 4, 2020 . [doi: 10.1101/2020.02.03.932350]

92. Chen X, Yao L, Zhang Y. Residual attention U-Net for automated multi-class segmentation of COVID-19 chest CT images. arXiv. Preprint posted online April 12, 2020.

93. Gaál G, Maga B, Lukács A. Attention U-Net based adversarial architectures for chest X-ray lung segmentation. arXiv. Preprint posted online March 23, 2020.

94. Huang L, Han R, Ai T, Yu P, Kang H, Tao Q, et al. Serial quantitative chest CT assessment of COVID-19: a deep learning approach. Radiol Cardiothoracic Imaging 2020 Apr 01;2(2):e200075. [doi: 10.1148/ryct.2020200075]

95. Shan F, Gao Y, Wang J, Shi W, Shi N, Han M, et al. Lung infection quantification of COVID-19 in CT images with deep learning. arXiv. Preprint posted online March 10, 2020.

96. Tang Z, Zhao W, Xie X, Zhong Z, Shi F, Liu J, et al. Severity assessment of coronavirus disease 2019 (COVID-19) using quantitative features from chest CT images. arXiv. Preprint posted online March 26, 2020 .

97. Yu H, Shao J, Guo Y, Xiang Y, Sun C, Yuan Y. Data-driven discovery of a clinical route for severity detection of COVID-19 pediatric cases. medRxiv. Preprint posted online March 20, 2020 . [doi: 10.1101/2020.03.09.20032219]

98. Bai X, Fang C, Zhou Y, Bai S, Liu Z, Chen Q, et al. Predicting COVID-19 malignant progression with AI techniques. medRxiv. Preprint posted online March 23, 2020 . [doi: 10.1101/2020.03.20.20037325]

99. DeCaprio D, Gartner J, McCall CJ, Burgess T, Kothari S, Sayed S. Building a COVID-19 vulnerability index. medRxiv. Preprint posted online March 21, 2020 . [doi: 10.1101/2020.03.16.20036723]

100. Gong J, Ou J, Qiu X, Jie Y, Chen Y, Yuan L, et al. A tool to early predict severe 2019-novel coronavirus pneumonia (COVID-19): a multicenter study using the risk nomogram in Wuhan and Guangdong, China. medRxiv. Preprint posted online March 20, 2020 . [doi: 10.1101/2020.03.17.20037515]

101. Pourhomayoun M, Shakibi M. Predicting mortality risk in patients with COVID-19 using artificial intelligence to help medical decision-making. medRxiv. Preprint posted online April 1, 2020 . [doi: 10.1101/2020.03.30.20047308]

102. Yan L, Zhang HT, Goncalves J, Xiao Y, Wang M, Guo Y, et al. A machine learning-based model for survival prediction in patients with severe COVID-19 infection. medRxiv. Preprint posted online March 17, 2020 . [doi: 10.1101/2020.02.27.20028027]

103. Sarkar J, Chakrabarti P. A machine learning model reveals older age and delayed hospitalization as predictors of mortality in patients with COVID-19. medRxiv. Preprint posted online March 30, 2020 . [doi: 10.1101/2020.03.25.20043331]

104. Qi X, Jiang Z, Yu Q, Shao C, Zhang H, Yue H, et al. Machine learning-based CT radiomics model for predicting hospital stay in patients with pneumonia associated with SARS-CoV-2 infection: a multicenter study. medRxiv. Preprint posted online March 3, 2020 . [doi: 10.1101/2020.02.29.20029603]

105. Pandey R, Gautam V, Bhagat K, Sethi T. A machine learning application for raising WASH awareness in the times of covid-19 pandemic. arXiv. Preprint posted online March 16, 2020 .

106. Rolling updates on coronavirus disease (COVID-19). World Health Organization. 2020. URL: https://www.who.int/ emergencies/diseases/novel-coronavirus-2019/events-as-they-happen [accessed 2020-05-21]

107. Konečný J, McMahan B, Ramage D. Federated optimization: distributed optimization beyond the datacenter. arXiv. Preprint posted online November 11, 2015 .

108. Kiwibot. 2020. URL: https://www.kiwibot.com/ [accessed 2020-05-22]

109. Whiteboard Coordinator. 2020. URL: https://wbcoordinator.com/ [accessed 2020-05-22]

110. EHTERAZ. App Store. 2020. URL: https://apps.apple.com/us/app/ehteraz/id1507150431 [accessed 2020-05-22]

\section{Abbreviations}

AI: artificial intelligence

CNN: convolutional neural network

CT: computed tomography

NCBI: National Center for Biotechnology Information

NLP: natural language processing

PRISMA-ScR: Preferred Reporting Items for Systematic Reviews and Meta-Analyses Extension for Scoping

Reviews

RNN: recurrent neural network

SVM: support vector machine 
Edited by G Eysenbach; submitted 27.05.20; peer-reviewed by N Fareed, KL Ong; comments to author 13.07.20; revised version received 26.07.20; accepted 29.07.20; published 15.12.20

Please cite as:

Abd-Alrazaq A, Alajlani M, Alhuwail D, Schneider J, Al-Kuwari S, Shah Z, Hamdi M, Househ M

Artificial Intelligence in the Fight Against COVID-19: Scoping Review

J Med Internet Res 2020;22(12):e20756

URL: http://www.jmir.org/2020/12/e20756/

doi: $10.2196 / 20756$

PMID: $\underline{3284779}$

(C)Alaa Abd-Alrazaq, Mohannad Alajlani, Dari Alhuwail, Jens Schneider, Saif Al-Kuwari, Zubair Shah, Mounir Hamdi, Mowafa Househ. Originally published in the Journal of Medical Internet Research (http://www.jmir.org), 15.12.2020. This is an open-access article distributed under the terms of the Creative Commons Attribution License (https://creativecommons.org/licenses/by/4.0/), which permits unrestricted use, distribution, and reproduction in any medium, provided the original work, first published in the Journal of Medical Internet Research, is properly cited. The complete bibliographic information, a link to the original publication on http://www.jmir.org/, as well as this copyright and license information must be included. 